\title{
Mathematics, signal, and computer background in an optical engineer education
}

Pierre Chavel, Jean Taboury

Pierre H. Chavel, Jean Taboury, "Mathematics, signal, and computer background in an optical engineer education," Proc. SPIE 3190, Fifth International Topical Meeting on Education and Training in Optics, (8 December 1997); doi: 10.1117/12.294404

Event: Fifth International Topical Meeting on Education and Training in Optics, 1997, Delft, Netherlands 
Mathematics, signal and computer background in an optical engineer education

\author{
P. Chavel and J. Taboury \\ Institut d'Optique Théorique et Appliquée, Ecole Supérieure d'Optique \\ B.P. 147, F 91403 Orsay cedex, France, e-mail pierre.chavel@iota.u-psud.fr, www.iota.u-psud.fr
}

\begin{abstract}
We have just redesigned the organization of courses in our Optical Engineering school. Mathematics was merged with Signal to emphasize the more generally useful concepts derived from Fourier analysis, sampling and convolution. Computers are introduced as tools with a practical understanding of systems based on training on several programming languages.
\end{abstract}

Keywords : education in optics, engineer, curriculum, mathematics, computer science, signal processing

\title{
1.INTRODUCTION
}

Our school delivers a degree of Optical Engineer. (The official title is Ingénieur Diplômé de l'Ecole Supérieure d'Optique ; the school is more widely known as SupOptique). As is traditional in French engineering education ${ }^{1}$, it is a five-year degree, but our students spend only the last three years in our school. The first two years of higher education are called the «preparatory classes » devoted to extensive training in mathematics, physics and chemistry with some basics of computer tools and engineering. These courses are offered in special classes, mostly in high schools. It is appropriate to mention here that after they have been admitted in an engineering school such as ours, students can well be expected to be fluent in all aspects of undergraduate mathematics, including linear algebra and integral and differential calculus.

The role of our school is therefore to give them the necessary specialized background to be employed as an optical engineer as soon as they graduate at age 23 to 25 , while of course providing them with the essential breadth of technical knowledge that will allow them to evolve as years pass : this includes in particular advanced training in physics and some familiarity with electronics. Of course, languages, business and communication are an additional part of the curriculum.

Every three to five years or so, some remodeling of the course structure is appropriate to keep with the evolution of science, technology. and economy. Committees including instructors, students, alumni and employers are then appointed to advise the academic director of the school before decisions are made. We just went through such a process for a new organization of courses to come in effect for the academic year 1997-98 and this communication relates to the choices that were made concerning mathematics, signal and computer science.

The users of the curriculum are the students, and the primary purpose in the present economic context is to help them find attractive positions as engineers. An analysis of the needs of potential employers is therefore essential. It is therefore appropriate to give some statistics about who these employers are ${ }^{2}$. They are almost evenly distributed into six categories : optics industry, electronics industry, defense, aerospace, research laboratories and miscellaneous. Half of the miscellaneous are in fact independent consultants, and the rest includes a growing but still small fraction of automotive and telecommunications. The job profiles of our alumni are heavily biased towards technical positions in research and development, while about $35 \%$ are in management, sales or miscellaneous positions. One noteworthy element is the relatively small fraction of those employed in optics industry stricto sensu. This is typical of a long trend of evolution from optics to a broader field, that includes optoelectronics 
and traditional optics and may be termed « photonics » to make it clear that optical instruments, albeit important, are by far not our only subject.

For a better understanding of the following discussion, it may be appropriate to mention some additional data about our system compared, for example, to US universities. Our system is not based on course credit capitalization and is organized by full academic years, not by semester. Course duration varies from topic to topic and in most cases instructors use no textbooks, although many of them handout their own lecture notes to the students. One academic year extends from early September until the last days of June with a total of six weeks of vacations during that period. In addition, students are requested to take laboratory internships outside the school at various points during the curriculum. Courses contents have evolved over the last thirty years or so to follow the evolution of the field of optics that was just mentioned. The net result is a clear increase in the number of exposure hours, that presently averages to a little over 900 hours, including ten percent devoted to written examinations. With some homework requested to study the courses and prepare various requested projects, we have reached the maximal tolerable load for an efficient education.

In the following, we discuss our analysis of the field of mathematics, signal and computers for theses students and list our new organization, that will come into effect in September 1997 for the first year students and propagate through the curriculum to be fully in effect in year 2000. It had been a prior decision that the total duration devoted to these matters was to remain constant at 220 exposure hours.

\section{ANALYSIS}

\section{Subjects covered}

Mathematics, signal theory and computer science were considered as a whole because coordination between them is required : a number of mathematical concepts are prerequisite for signal theory and signal processing, exercises that can illustrate and refine signal theory concepts are best done on computers, and therefore the instructors must have an efficient coordination. On the other hand, even though there may be exceptions, our students are not intended to be future specialists in those domains. Therefore, they have to be introduced as tools for a better education in photonics and restricted to this purpose.

\section{From mathematics to signal}

In an attempt at defining the mathematics courses topics that were required for some optics and photonics courses at some stage or another during the three years curriculum and were not included in the previous education of our students, the following list was identified :

- Hilbert spaces (for quantum mechanics)

- Bessel functions of integer order (for diffraction, optical design, modulation and guided wave optics)

- Orthogonal polynomials : Hermite, Laguerre, Legendre, Zernike (for laser modes and optical transfer functions)

- Analytical functions, integration in complex plane (as a general tool to compute integrals in various domains)

- Tensor calculus (for nonlinear optics, acousto-, magneto- and electro-optics)

- Fourier transforms and linear filtering (as a general tool for signal and image processing, electronics, diffraction, Fourier optics, optical design, spectrometry, detectors as well as quantum mechanics)

- Laplace transforms (for electronic linear filtering and control)

- Green functions (for electromagnetism) 
- Probability, random variables and stochastic processes (as a general tool for signal and image processing, detectors, coherence, quantum mechanics)

- Mathematical methods for nonlinear physics : chaos, attractors, fractals (for nonlinear optics and laser dynamics).

- Introduction to numerical analysis (as a general tool for physics and signal processing).

One guideline for dealing with these fairly unrelated topics came from our past experience : there appeared to exist a lack of motivation of students whenever there was a long delay between the time a concepts of mathematics in signal is introduced and the time it is useful for an optics or more general physics related course. In addition, several of the above topics are not closely related. This argues in favor of asking each instructor to introduce the students to whatever mathematical tools are needed for him.

This policy, however, cannot apply to topics that are needed in several courses. These have to be introduced earlier than the first course that needs them and the students need to spend enough time on exercises that they become familiar with the concepts. Looking at the previous list, this comment applies clearly to one domain only, namely the theory of linear deterministic or stochastic systems, including Fourier and Laplace transforms. Nevertheless, whether mathematical concepts useful for a given chapter of physics should always be introduced before the physics is not as obvious as it appears, since some more theoretical subjects like rigorous introductions of distributions or of probability theory may obscure the issue to the unprepared students rather than pave the way to a better understanding of physics courses. Such more advanced subjects should preferably be considered, if time permits, as a concluding synthesis that will improve the confidence of students in the consistency of the mathematics behind subjects that will have been introduced in a partially intuitive way.

One final note of general relevance is that the very name of mathematics hints at a rigorous approach ; to some students, rigorous means dull while some others are more mathematically minded and will be disappointed at any course that is not really rigorous; yet, a rigorous introduction, for example, of the Fourier analysis of stochastic processes would be completely irrealistic given our time constraints. It is a fact also that formal courses of mathematics like we used to offer years ago with extended introductions of integration and measure theories and of tensor algebra are no more compatible with our time constraints. We therefore decided to give up the word « Mathematics » altogether in our course list, to introduce « Signal » instead as a consistent body of knowledge of broad interest to many courses taught in our school, and to ask the instructors needing any other topic in the above list to introduce it themselves together with the application.

\section{Computer science and computer tools}

The objective with the courses on computer science and computer tools are not to educate computer scientists, but to familiarize students with some important tools for engineers : the concept of a computer system, the concept of a computer, basic operations on present computers, programming and algorithmics. Again, these concepts should be introduced in a practical rather than formal way.

The problem when considering programming in a practical way is that programming languages are needed, there exists a large variety of them and it is a heavy investment to master the peculiarities of any one of them. In our previous organization, we started with Pascal because students had been introduced to it in their preparatory classes (see section 1). Our initial idea with this was that students would be familiar with Pascal and could more easily concentrate on more basic issues in programming; this did not prove true : unlike in algebra and calculus where the dispersion between our first year students is fairly small, there was a considerable dispersion in their familiarity with Pascal. In addition, while Pascal is a good tool from a didactic point of view, it is hardly used anywhere after school nowadays. However, changes were introduced in the national regulations for preparatory classes and Pascal has 
now been abandoned; it has been replaced by formal manipulation tools such as Mathematica(®) or Maple(®). Abandoning Pascal was therefore an easy decision, and we did not think that continuity in exposure to Mathematica or Maple was needed for our students. This, nevertheless, did not solve the problem of which language to teach. In our discussions with employers, we were confronted with two opposite viewpoints : for some employers, even though our students will be employed as optical engineers, they still need to master programming in itself for general purpose use in a business environment. For some others, they should be able to use programming tools for scientific applications. In addition, skills in computers of ten have one major weight when selecting among applicants for a job. After pondering with the various arguments, we felt that we were not a position to select less than three languages for our curriculum.

- Matlab(®) will be introduced as a modern tool for engineers that has powerful features for scientific calculation and offers extended programming possibilities. It is our experience that this language tends to spread among the junior alumni and that students exposed to it like very much using it. Also, since at present virtually no student enters our school with a previous knowledge of Matlab, this makes the starting phase of computer classes easier because all students are nearly at the same level.

- Fortran will keep its right as a long lived language widely developed in scientific programming and whose standards evolve as computer science progresses.

- Finally, for general purpose programming, $\mathrm{C}++$ was selected as the most popular solution among potential employers.

This general backbone of computer classes will be briefly introduced by notions about the organization of the school's computer system and the use of computers as office tools, since not all new students know that a word processor differs from a typewriter and that you do not need to use your pocket calculator to fill in the cells of a spreadsheet. In addition, some computer software tools specifically designed for optical scientists are introduced as part of optics courses on optical design, illumination and photometry, wave propagation, and others.

\section{OUR PRESENT SOLUTION}

Instead of a conclusion, we shall close this communication with the course description as it results from the above considerations. It is appropriate to note that, as soon as financial issues are cleared, all computer equipment available to the students will consist of Pentium PC computers and PowerPC Macintoshes.

\section{Signal theory (first year)}

Signal processing and signal theory are introduced as a scientific field in its full right, which is necessary on a daily basis to engineers and needed in many other courses at SupOptique (physical optics, electronics and control, image processing, noise, optics and electronics laboratory courses). This field is therefore taught as an extended introduction throughout the first year of the curriculum that covers deterministic and stochastic signals as well as continuous and discrete signals. Emphasis is laid on spectral analysis (Fourier Transform) with a short, intuitive presentation of the use of distribution, and on filtering and convolution. The total extends over 60 hours lectures and 17 hours exercise courses, plus 9 hours written examinations. It is decomposend in three parts of equal weight :

- Deterministic signals

- Stochastic signals

- Characterization and processing of stochastic signals 
Image processing (third year)

An introductory course on image processing covers image sampling concepts and equipment, image enhancement, image restoration, segmentation and classification. 18 hours lectures plus 2 hours written examination.

Computers (first year)

Computers at SupOptique ; the Matlab tool and programming language, exercises in numerical analysis of signals. 36 hours lectures and exercise classes. The examination takes the form of a homework project, with the instructor being available at given hours to advise the students on their project.

\section{Computers (second year)}

Algorithmics and programming in Fortran 90. Exercises on Fourier transformation and filtering. 54 hours lectures and exercise classes. Same kind of examination as in the first year.

Computers (third year)

Programming in $\mathrm{C}$, object language programming, the $\mathrm{C}++$ programming language. Exercises on general purpose programming. 36 hours lectures and exercise classes. Same kind of examination as in the first two years.

\section{ACKNOWLEDGEMENTS}

We thank our colleagues M. Allain, M. Bénidir, F. Bernard, J.F. Cabadi, F. Forest, N. Kebaïli, A. Marion, R. Mercier, H. Sauer and Y. Tanguy, who will participate in teaching these courses. Together with several SupOptique alumni and students, they have contributed redesigning the mathematics, signal and computer part of the curriculum at our school as described in this manuscript.

\section{REFERENCES}

${ }^{1}$ P. Chavel, C. Froehly, «Optical Education and Training in France », International Conference on Optics Education, G. Altshuler and B.J. Thompson, editors, SPIE Proc. Vol. 1603, pp 41-47, SPIE, Bellingham, 1992.

2 Association Amicale des Anciens Elèves de l'Ecole Supérieure d'Optique, « Annuaire 1996 », Orsay, 1996 (annual membership list of the Alumni Association). 